\title{
Memory in the pigeon: Proactive inhibition in a delayed matching task ${ }^{*}$
}

\author{
THOMAS R. ZENTALL and DAVID E. HOGAN \\ University of Pittsburgh, Pittsburgh, Pennsylvania 15260
}

\begin{abstract}
Proactive inhibition in a pigeon was studied, using a modified delayed matching-to-sample task. Proactive inhibition was defined as disruption in matching performance produced by the presentation of a stimulus prior to the sample stimulus (relative to control trials without prior stimulus presentation). In Experiment I, with a prior stimulus which was not presented as one of the comparison stimuli, temporary disruption in matching performance was found with a 5 -sec delay between sample and comparison stimuli but not with a 0-sec delay. In Experiment II, with a prior stimulus which was later presented as the incorrect comparison stimulus, long-lasting disruption was found with a 5-sec delay, but again not with a 0 -sec delay. Experiment III demonstrated a direct relation between the magnitude of proactive inhibition and length of the delay. These results offer some support for an interference theory of forgetting, but also suggest that it may not be the identity of the two stimuli but, rather, the order of the two stimuli which is lost.
\end{abstract}

The study of memory in animals has been plagued by the question of whether the measure of memory loss is at least partially confounded with failure to instruct the animal at the time of test. This is particularly true when one is using a prior task (proactive inhibition) or interpolated task (retroactive inhibition) to disrupt memory (see Zentall, 1970).

Zentall (1973), using a within-S procedure, separated instruction learning from the measurement of short-term memory loss in pigeons with the use of a delayed matching-to-sample task. Forgetting was manipulated by varying stimulus presentation during the retention interval. The series of events involved in the delayed-matching procedure is analogous to a control condition against which memory loss in the retroactive inhibition paradigm can be evaluated, i.e., a single task (pecking the sample), followed by retention interval and test. Zentall (1973) alternated standard delayed-matching trials with experimental trials, which involved stimulus presentation during the delay between sample and comparison stimuli. One can think of interpolated stimulus presentation as being a second task, even though responses to it were not required, nor did they have any effect on the sequence of events. The interpolated stimuli never appeared as comparison stimuli, which means that at the time of test there could have been no confusion as to which of the two prior stimuli was the sample; only one of the two had been presented. Yet, Zentall (1973) observed significant disruption of delayed matching performance on interpolated-stimulus trials, indicating the presence of

*This research was supported in part by Biomedical Sciences Support Grant FR07084 from the National Institu tes of Health to the University of Pittsburgh, and by National Institute of Mental Health Grant MH19757 to the first author. We thank Karen Compomizzi, Celeste Compomizzi, Nancy Threlfall, and Patricia Hughes for their assistance with experimental sessions. Reprints may be obtained from T. R. Zentall, Department of Psychology, University of Pittsburgh, Pittsburgh, Pennsylvania 15260. retroactive inhibition in the absence of an instructional problem.

The purpose of the present studies was to test for the presence of proactive inhibition in the pigeon, using procedures similar to those of Zentall (1973), and in addition, to test a prediction of the interference theory of forgetting (Postman \& Underwood, 1973), namely that the magnitude of proactive inhibition should increase as the delay increases between second-task learning and second-task retention test. This prediction is based on the assumption that (1) Task 1 responses extinguish during the learning of Task 2, and (2) Task 1 responses recover over time, due to spontaneous recovery, compete with Task 2 responses, and result in increased proactive inhibition.

The present studies also sought to test the hypothesis that an increase in proactive inhibition over time may result not from memory loss for the second task, but rather from memory loss for the order of the two tasks, i.e., which of the two tasks was performed last (Gleitman, 1971; Spear, 1971; Zentall, 1970).

\section{EXPERIMENT I}

To measure proactive inhibition, Zentall's (1973) procedure was modified such that stimuli were presented prior to the sample stimulus rather than following the sample stimulus. In order to insure that the interval between Task 1 and test was held constant, Zentall (1973) required no response to the interpolated stimulus. But the absence of a response to the interpolated stimulus made it possible for the bird to avoid perception of the interpolated stimulus by looking away from the key. The fact that the significant disruption found by Zentall (1973) dissipated over trials may have been due to the pigeon's poorer reception of the interpolated stimulus, rather than better processing, storage, or retrieval of the sample. With stimulus 


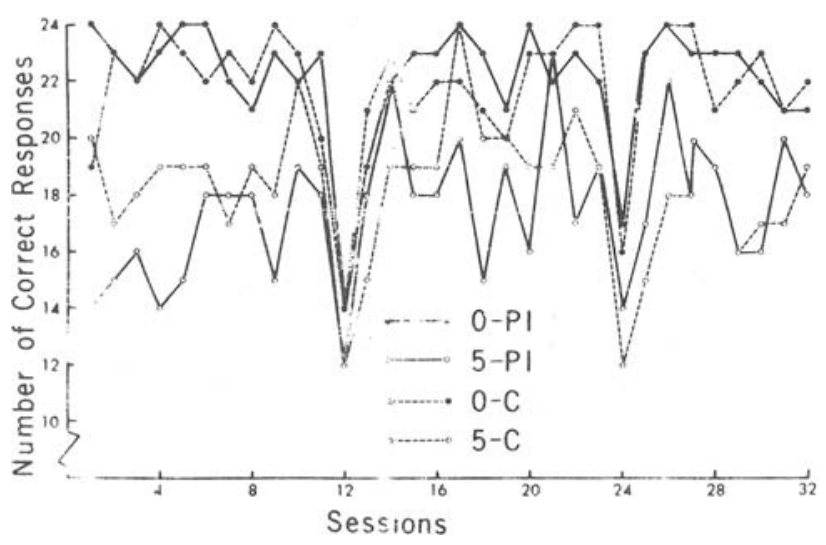

Fig. 1. Number of correct responses for each experimental session of Experiment I for proactive inhibition 0-sec-delay (0-PI) and 5-sec-delay (5-PI) trials, and control 0-sec-delay (0-C) and 5-sec-delay (5-C) trials. On a PI trial the prior stimulus never appeared as one of the comparison stimuli.

presentation prior to the sample, however, one can require responding to the inhibiting stimulus and still hold constant the interval between the sample and the comparison stimuli. In the present study, responding to the prior stimulus was required before the sample stimulus was presented.

Additionally, with Zentall's (1973) procedure, the interpolated stimuli were never presented as comparison stimuli, either within trials or across trials. In the present procedure, the prior stimulus was never presented as a comparison stimulus within a trial, but it often appeared as either the sample or the incorrect comparison stimulus on other trials.

\section{Method}

Subject. One female White Carneaux pigeon, approximately 4 years old, which had had extensive delayed matching-to-sample training, was maintained at $75 \%-80 \%$ of her free-feeding weight.

Apparatus. A standard three-response-key pigeon test chamber was used. Behind each key was an in-line projector which projected a red, green, or blue field onto the key. A dim hou selight was on at all times except during the intertrial interval (ITI). Reinforcement consisted of 3-sec access to Purina pigeon grain. External sound masking was produced by white noise and a blower fan.

Procedure. Five sessions of baseline training were given. Each trial began with the onset of the center (sample) key. Five responses to the sample turned it off and initiated a delay. The end of the delay determined the onset of the side (comparison) keys. A response to the comparison key which matched the sample was reinforced and initiated a 5-sec ITI, while a response to the other key merely initiated the ITI. Red, blue, and green stimuli served as the sample and comparison stimuli. Each baseline session consisted of 480 -sec delay (simultaneous offset of sample and onset of comparison stimuli), and 48 5-sec delay randomly alternating trials.

Color of the sample, color of the "odd" comparison stimulus, and position of the matching comparison stimulus were counterbalanced over each 96-trial session.

Following baseline training, 32 daily experimental sessions $(96$ trials each) were run. These sessions consisted of four kinds of trials, equally represented: 0-sec-delay control trials and 5-sec-delay control trials as during baseline training, and 0-sec-delay proactive inhibition (PI) trials and 5-sec-delay PI trials. On PI trials, the prior stimulus appeared on the center key at the start of the trial and five responses replaced the prior stimulus with the sample. Thus, not until after the fifth response was information provided to the pigeon as to whether the first stimulus was a prior stimulus or a sample. In all other respects, PI and control trials were the same. The order of trial presentation was random, with the constraint that no more than three consecu tive trials were of one kind.

\section{Results}

Data from baseline sessions indicated a high level of mean performance on both 0 -sec-delay trials $(\overline{\mathrm{X}}=44.2$, $92 \%$ correct $)$ and 5 -sec-delay trials $(X=41.4,86.2 \%$ correct).

The data from experimental sessions are presented in Fig. 1. Five-second-delay performance was initially disrupted by the prior stimulus, but after about five sessions little disruption remained. If one considers performance on each session as an independent sample of this particular pigeon's performance, one can perform a two-way analysis of variance on these scores. Such an analysis performed on scores for the first five sessions indicated a significant PI effect, $F(1,16)=6.0, p<.05$, a significant delay effect, $F(1,16)=122.4, p<.01$, and a significant PI by Delay interaction, $F(1,16)=18.1$, $\mathrm{p}<.01$. The interaction indicates increasing PI as a function of retention interval. Over sessions, the analysis indicated a significant delay effect, $F(1,128)=105.4$, $\mathrm{p}<.01$, but no PI effect, $\mathrm{F}<1$, and no significant PI by Delay interaction, $\mathrm{F}(1,128)=2.29, \mathrm{p}>.05$.

Mean correct responses over experimental sessions was $21.8(90.8 \%$ correct) and 22.1 (92.2\% correct) for 0-sec-delay control and PI trials, respectively, and 18.2 (75.8\% correct) and 17.4 (72.5\%) correct for 5-sec-delay control and PI trials, respectively.

Disrupted performance on Session 12 may have been due to a burned-out houselight lamp which was not discovered until the session was complete. Similar disruption on Session 24 cannot be explained.

\section{EXPERIMENT II}

Since only minimal disruption of delayed matching performance was found when the prior stimulus never appeared as one of the comparison stimuli, it was decided to determine the effects of presentation of the prior stimulus as the incorrect comparison stimulus. But presentation of the prior stimulus during test allows for the possibility that disruption of performance by prior stimulus presentation might be due to the absence of instructions (i.e., information indicating which of the stimuli presented on the center key was the sample), rather than memory loss of the sample. Instructions should have been well learned in Experiment I, however, due to extensive practice with prior stimuli. The 32 experimental sessions in Experiment I can be thought of as training with the instruction, "Respond to the stimulus last seen." More importantly, though, the present study allows for the independent assessment of 
memory loss, since lack of instructions should affect disruption equally at both the 0 -sec delay and 5-sec delay. Any difference therefore between prior stimulus disruption at 0 -sec delay and 5-sec delay can be attributed to memory loss rather than lack of instructions.

\section{Method}

The same bird and apparatus used in Experiment I were used in Experiment II. Sessions were the same as those in Experiment I, with the exception that during PI trials the color presented as the prior stimulus was also presented as the incorrect comparison stimulus on that trial. This procedure required memory for not only which of the two comparison stimuli had been seen on the center key earlier during the trial, but also which of the two comparison stimuli had been last seen. Experiment II consisted of 34 daily sessions.

\section{Results}

Data from Experiment II are presented in Fig. 2. The data indicate pronounced and prolonged proactive inhibition on 5-sec-delay trials, and little or no proactive inhibition on 0-sec-delay trials. A two-way analysis of variance performed on the scores for each session indicated a significant PI effect, $F(1,132)=127.7$, $\mathrm{p}<.01$, a significant delay effect, $\mathrm{F}(1,132)=445.4$, $\mathrm{p}<.01$, and a significant PI by Delay interaction, $F(1,132)=49.2, p<.01$. The interaction indicates increasing PI as a function of retention interval.

Mean number of correct responses averaged over sessions were 23.0 (96.6\% correct) and 21.9 (91.3\% correct) for 0-sec-delay control and PI trials, respectively, and 19.3 (80.5\% correct) and 14.3 (59.6\% correct) for 5-sec-delay control and PI trials, respectively.

\section{EXPERIMENT III}

Experiment III was an attempt to explore, in more detail, the relation between prior stimulus presentation

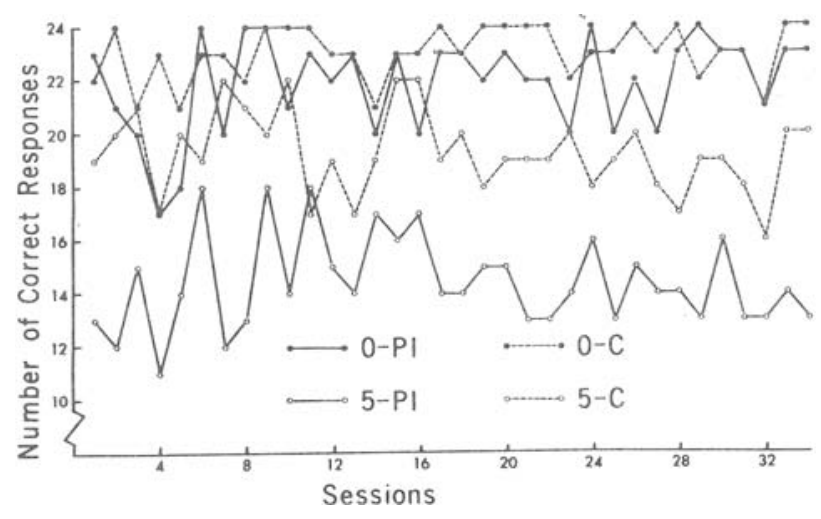

Fig. 2. Number of correct responses for each session of Experiment II, for proactive inhibition 0-sec-delay (0-PI) and 5-sec-delay (5-PI) trials, and control 0-sec-delay (0-C) and 5-sec-delay (5-C) trials. On a PI trial, the prior stimulus always appeared as one of the comparison stimuli.

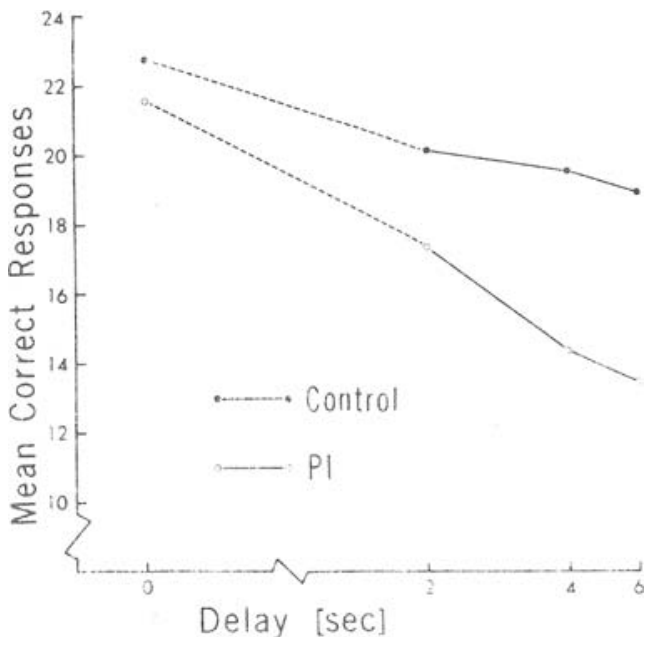

Fig. 3. Mean performance pooled over sessions for PI and control trials, at each delay. During all sessions, the prior stimulus on a PI trial always appeared as one of the comparison stimuli.

and retention interval. In Experiment III, four retention intervals were studied.

\section{Method}

The S and apparatus were the same as in Experiments I and II. On PI trials, as in Experiment II, the prior-presented stimulus always appeared as one of the comparison stimuli. For the first 5 sessions (baseline), 0-sec-delay and 6-sec-delay trials were run, to insure familiarity with slightly longer delay. The remaining 24 sessions involved 6 blocks of 4 sessions each, in an ABCCBA design with 0-sec-delay and 2-sec-delay trials run on the first and sixth blocks, 0 -sec-delay and 4-sec-delay trials run on the second and fifth blocks, and 0-sec-delay and 6-sec-delay trials run on the third and fourth blocks.

\section{Results}

Performance on baseline sessions was quite similar to performance in Experiment II. Mean number of correct responses averaged over the 5 sessions were 23.4 (97.5\% correct) and $21.8(90.8 \%$ correct $)$ for 0 -sec-delay control and PI trials, respectively, and $19.2(80.0 \%$ correct $)$ and 14.4 (60.0\% correct) for 6-sec-delay control and PI trials, respectively.

Data from experimental sessions are presented in Fig. 3. The trend in relation between length-of-delay and degree of disruption can clearly be seen. A two-way analysis of variance performed on the scores for each experimental session indicated a significant PI effect, $\mathrm{F}(1,88)=51.6, \mathrm{p}<.01$, a significant delay effect, $F(3,88)=51.2, p<.01$, and a significant PI by Delay interaction, $F(3,88)=6.9, p<.01$. The linear trend of the PI by Delay interaction was also significant, $F(1,88)$ $=15.4, \mathrm{p}<.01$, indicating that longer retention intervals produced more PI.

\section{GENERAL DISCUSSION}

The results of the present studies confirm the existence of proactive inhibition in the pigeon, and indicate that this effect 
behaves in an orderly fashion. Prior presentation of a stimulus not presented at the time of test had a temporary disruptive effect on 5-sec-delay matching trials, while prior presentation of the incorrect comparison stimulus had a prolonged disruptive effect on 5-sec-delay matching trials. These results offer support for interference theory, which predicts increasing proactive inhibition as the "extinguished" prior stimulus has the opportunity to recover spontaneously. But the fact that long-lasting proactive inhibition was demonstrated only in Experiments II and III suggests that the pigeon probably forgot the order in which the two stimuli were presented rather than the identity of the two colors seen. If prior stimulus presentation produced a memory loss for the sample stimulus, then delayed performance on PI trials should not have been poorer when the prior stimulus was presented during test than when it was absent. Since performance was poorer when the retention test involved the prior stimulus, the pigeon could apparently remember that it had seen the sample but had difficulty remembering whether it had been seen first or second.

\section{REFERENCES}

Gleitman, H. Forgetting of long-term memory in animals. In W. K. Honig and P. H. R. James (Eds.), Animal memory. New York: Academic Press, 1971.

Postman, L., \& Underwood, B. J. Critical issues in interference theory. Memory \& Cognition, 1973, 1, 19-40.

Spear, N. E. Forgetting as retrieval failure. In W. K. Honig and P. H. R. James (Eds.), Animal memory. New York: Academic Press, 1971.

Zentall, T. R. Effects of context change on forgetting in rats. Journal of Experimental Psychology, 1970, 86, 440-448.

Zentall, T. R. Memory in the pigeon: Retroactive inhibition in a delayed matching task. Bulletin of the Psychonomic Society, 1973, 1, 126-128.

(Received for publication April 26, 1974.) 